\title{
HIPÓFISIS Y SUPRARRENALES
}

\section{Póster}

\section{2 semanas de tratamiento con análogos de somatostatina: respuesta bioquímica y tamaño tumoral en pacientes con acromegalia}

\author{
Abreu A, Osorio CV, Salgado CA, Ramírez A, Barakat S, \\ Carvajal $R$.
}

Universidad Libre, Centro Médico Imbanaco. Cali, Colombia.

$\mathrm{E}$ l uso de análogos de somatostatina (AS) en acromegalia tiene como objetivos reducir el tamaño tumoral y normalizar los niveles del factor de crecimiento (GH) y la somatomedina C (IGF-1).

Se reporta una serie de casos retrospectivo de pacientes con acromegalia que recibieron AS entre 2015 y 2017 . Mediante el análisis de varianza de medidas repetidas, se analizaron las diferencias de GH y IGF-1 basal y a las 24 y 52 semanas, el tamaño tumoral inicial y a las 52 semanas postratamiento entre lanreotida y octreotida.

29 pacientes entre 28 y 79 años completaron 52 semanas de seguimiento. Se expresan en valor medio el tamaño tumoral en hombres: $16 \mathrm{~mm}$ y en mujeres: $19 \mathrm{~mm}, \mathrm{GH}$ en hombres y mujeres: $13 \mathrm{ng} / \mathrm{mL}$, IGF-1 en hombres: $773 \mathrm{ng} / \mathrm{mL}$ y en mujeres: $780 \mathrm{ng} / \mathrm{mL}$.

El valor de GH disminuyó desde el inicio $(13,5 \mathrm{ng} / \mathrm{mL} \pm$ 1,7) con intervalo de confianza (IC) $95 \%$ : 10,0-17,0, y en la semana $52(2,2 \mathrm{ng} / \mathrm{mL} \pm 0,3)$ con IC $95 \%$ : 1,6-2,7 $(p=0,000)$. La IGF-1 inicial fue de $776,7 \mathrm{ng} / \mathrm{mL} \pm 41,3$ con IC $95 \%$ : 692,2861,3 , y en la semana 52 fue de $336,9 \mathrm{ng} / \mathrm{mL} \pm 29,0$ con IC 95 $\%: 277,3-396,5(p=0,000)$. La media de GH con lanreotida y octreotida a la semana 24 fue de 4,72 ng/mL \pm 5,1 con IC 95\%: 1-7,4 frente a $5,2 \mathrm{ng} / \mathrm{mL} \pm 4,5$ con IC $95 \%:-0,43$ y $-10,9$, respectivamente ( $p=0,125)$; a la semana 52 fue de $2,4 \mathrm{ng} / \mathrm{ml} \pm$
1,3 con IC 95\%: 1,7-3,1, frente a $2,7 \mathrm{ng} / \mathrm{mL} \pm 1,5$ con IC $95 \%$ : $0,3-5,1(p=0,467)$. La media del tamaño tumoral inicial con lanreotida y octreotida fue de 17,1 mm con IC $95 \%$ : 13,7 y 20,5 , frente a 14,6 mm con IC 95\%: 8,1-21,1 ( $p=0,431$ ); el tamaño final fue de 6,8 mm con IC $95 \%$ : 4,7-8,8 frente a 6,2 mm con IC $95 \%$ : 4,2-8,2 ( $p=0,759)$.

En conclusión, la remisión bioquímica y la disminución del tamaño tumoral a la semana 24 y 52 fue significativa en pacientes con acromegalia que recibieron manejo con AS sin tener diferencias entre los 2 medicamentos.

\section{Póster \\ Carcinoma adrenocortical con ACTH elevada: reporte de caso}

Fierro $F^{1}$, Fuentes $O^{2}$, Coy $A F^{3}$.

${ }^{1}$ Unidad de Endocrinología, Instituto Nacional de Cancerología. Bogotá D. C., Colombia.

${ }^{2}$ Unidad de Endocrinología, Hospital Universitario San Ignacio. Bogotá D. C., Colombia.

${ }^{3}$ Unidad de Endocrinología, Hospital San José. Bogotá D. C., Colombia.

Introducción: para el enfoque diagnóstico del hipercortisolismo, la medición de corticotropina (ACTH) sérica es un instrumento de alta utilidad porque permite diferenciar la etiología suprarrenal de la hipofisaria. Sin embargo, su utilidad depende tanto de las características operativas de la prueba diagnóstica como de su reproducibilidad en distintas instituciones. Se presenta un caso de ACTH con falso positivo y las dificultades diagnósticas derivadas del mismo.

Descripción del caso: paciente de 42 años de edad de sexo femenino con cuadro clínico de 1 año de evolución consistente en el aumento de $20 \mathrm{~kg}$ de peso e hipertensión arterial incidente, quien ingresó a nuestra institución con un diagnóstico de carcinoma adrenocortical con ACTH elevada en 2 
muestras y resonancia magnética cerebral dentro de los parámetros normales. Se consideró la posibilidad de síndrome de Cushing ectópico frente a un error de patología.

Desenlace clínico: se repitió la lectura de la patología, se solicitó una nueva muestra de ACTH y se encontró que estaba suprimida, lo que concordó con la etiología de carcinoma adrenocortical y se confirmó un falso positivo inicial. Se inició el manejo dirigido con mejoría de tensión arterial y se continuó el protocolo de titulación de mitotano.

Discusión y conclusiones: los falsos positivos en las ayudas diagnósticas pueden llevar a impresiones diagnósticas equívocas que afectan el manejo clínico. Se describe un caso de hipercortisolismo con discordancia entre el cuadro clínico y las pruebas bioquímicas. La comunicación entre el médico y el laboratorio, así como la investigación apropiada (incluida la dilución de la muestra, el uso de anticuerpos bloqueantes y las pruebas en plataformas alternativas) pueden contribuir a mejorar el enfoque diagnóstico y generar mejores desenlaces.

\section{Póster}

\section{Diabetes no controlada, Cushing incidental. Reporte de caso}

Jaramillo A, Mejía $H$.

Hospital Universitario Fundación Santa Fe de Bogotá, Colombia.

$\mathrm{S}$ e reporta a una paciente femenina de 68 años diabética tipo 2 desde 1985, en tratamiento con insulina (1987) y cambio a análogos de segunda generación y rápidas en 2015, con presencia de alta variabilidad, múltiples episodios de hipoglucemia y mal control metabólico, por lo que en 2016 se inició un sistema de microinfusión de insulina VEO 640 y se logró un control parcial, con hemoglobina glucosilada $\left(\mathrm{HbA}_{1 \mathrm{c}}\right)$ entre 8 \% y 8,5 \%. La paciente persistió con alta variabilidad y en diciembre de 2018 presentó cetoacidosis diabética sin causa desencadenante; ante el deterioro neurológico se solicitó una tomografía axial computarizada (TAC) cerebral que demostró un macroadenoma de hipófisis, confirmado con resonancia (masa en adenohipófisis del lado izquierdo de $14 \mathrm{~mm}$ ). Se realizó un perfil hipofisario con un aumento del cortisol libre en la orina de 24 horas: $534 \mu \mathrm{g} / \mathrm{mL}$ (valor de referencia [VR]: 150-190) y volumen de 3160 cc; el resto del perfil hipofisario fue normal. Además, se encontraron los campos visuales con alteración de la fibra nerviosa en ambos ojos.

Se hospitalizó para realizar el manejo quirúrgico del adenoma y la confirmación de la hipercortisolemia. El cortisol después de $1 \mathrm{mg}$ de dexametasona en 384,6 nmol/L (13,9 $\mu \mathrm{g} /$ dL), la ACTH en 74 pg/mL (VR: 4,7-48). La paciente se llevó a procedimiento quirúrgico el 17 de marzo de 2019, con el siguiente reporte de patología: macroadenoma de hipófisis productor de ACTH, subtipo escasamente granulado, Ki67 del $5 \%$ y reactividad intensa para la citoqueratina CAM 5,2. Después del procedimiento se logró el descenso rápido de los requerimientos de insulina, posiblemente con el retiro del sistema de microinfusión de insulina prontamente.

El síndrome de Cushing es una condición infrecuente, pero que debe descartarse en pacientes diabéticos que persisten con mal control metabólico y altos requerimientos de medicamentos.

\section{Póster}

\section{Fiebre de origen desconocido (FOD) secundaria a feocromocitoma}

\author{
González $A M^{1,2}$, Fierro $L F^{2}$, Muñoz $J D^{1}$, Bermúdez $L N^{1}$, \\ Guzmán JD', Vanegas $J J^{1}$.
}

${ }^{1}$ Universidad Nacional de Colombia.

${ }^{2}$ Instituto Nacional de Cancerología.

Introducción: el feocromocitoma es una neoplasia generalmente benigna de las células cromafines propias de la médula suprarrenal, caracterizado por producir grandes cantidades de catecolaminas, aunque con capacidad de secretar péptidos biológicamente activos como citocinas, principalmente interleucina 1 (IL-1), interleucina 6 (IL-6) y factor de necrosis tumoral alfa (TNF- $\alpha$ ).

Presentación del caso: se describe un paciente de 24 años quien consultó por picos febriles superiores a $39{ }^{\circ} \mathrm{C}$ intermitentes, pero diarios de 8 días de evolución asociados con mialgias y coluria. Excepto por la fiebre, el examen físico fue normal. En los paraclínicos se evidenció leucocitos, plaquetas, transaminasas y proteína C-reactiva (PCR) elevadas. Se descartaron causas infecciosas y autoinmunes. La radiografía de tórax fue normal y ecocardiograma resultó sin vegetaciones, pero en la ecografía abdominal se observó una masa suprarrenal izquierda de 7 x $5 \mathrm{~cm}$. Debido a estos hallazgos, se sospechó de una fiebre de origen tumoral, por lo cual se realizó una prueba terapéutica con naproxeno con buena respuesta y se practicó una tomografía por emisión de positrones-tomografía computarizada (PET-CT) con fluorodesoxiglucosa (FDG) para descartar metástasis, con el hallazgo de una captación exclusiva en la masa adrenal. Se evidenciaron metanefrinas diferenciadas elevadas en la orina, cortisol basal elevado que no suprime con dexametasona y corticotropina (ACTH) elevada. Se 
llevó a resección tumoral y se resolvió todo el cuadro clínico. El reporte de patología informó de un feocromocitoma.

Conclusión: el feocromocitoma puede ser una causa de síndrome febril con elevación de marcadores de inflamación. La IL-6 parece ser el mediador principal, el cual también explicaría las elevaciones en el cortisol al estimular en la hipófisis la producción de ACTH. Este tumor inusual debe incluirse en la larga lista de causas de FOD.

Palabras clave: feocromocitoma, fiebre de origen desconocido, interleucina 6.

\section{Póster}

\section{Hallazgos de patología suprarrenal en autopsias en el departamento de patología del Hospital Universitario de Santander, 2012-2018}

\section{Mantilla AF, Lozano JF, Padilla LE, Mantilla JC.}

Introducción: las alteraciones de la glándula suprarrenal originan desbalances corporales importantes que pueden llevar incluso a la muerte. Se clasifican en 3 grandes grupos: alteraciones estructurales, infecciosas y necrohemorrágicas. Este estudio describe la prevalencia post mortem de patología suprarrenal, que brinda al clínico una visión del panorama local en pacientes con desenlaces fatales, como herramienta que le permita enfocar el abordaje de este órgano.

Metodología: estudio observacional descriptivo de corte transversal de los protocolos de autopsias realizadas en el departamento de patología del Hospital Universitario de Santander entre el año 2012 y 2018, en el que se identifican a aquellos con patología suprarrenal. Se registró información sociodemográfica, de la patología y comorbilidades.

Resultados: se encontraron 97 autopsias con patología suprarrenal, la edad media fue de 39,54 $( \pm 19,54)$ años en nacidos vivos y $26,93( \pm 6,96)$ semanas en no nacidos. El $60,55 \%$ corresponde a hombres. La patología infecciosa $(47,42 \%)$ fue el principal hallazgo, incluidos el citomegalovirus (34,78\%), tuberculosis $(19,56 \%)$ e histoplasmosis (17,39 \%). Dentro de las alteraciones estructurales (31,95\%) se encontraron como principales hallazgos la metástasis (35,48\%) e hipoplasia (29,03 \%). Finalmente, el 20,61 \% presentó patología necrohemorrágica. La principal comorbilidad fue el virus de inmunodeficiencia adquirida (VIH; $36,08 \%$ ) y en un $17,52 \%$ de los casos la patología suprarrenal tuvo relación directa con la causa de muerte.
Conclusiones: dentro de la patología suprarrenal, las enfermedades infecciosas son las de mayor importancia epidemiológica, se encuentran especialmente asociadas con citomegalovirus y tuberculosis, y es fundamental considerar su alteración y abordaje entre la población con VIH, al ser la comorbilidad encontrada con mayor frecuencia.

\section{Póster \\ Hipofisitis por IgG4: reporte de un caso}

\author{
Guzmán $G^{1,2}$, Hormaza $A^{2,3}$, Martínez $V^{1,3}$, Ortega $S^{2}$, \\ Ortiz $D^{2}$.
}

${ }^{1}$ Departamento de Endocrinología, Fundación Valle del Lili. Cali, Colombia. ${ }^{2}$ Universidad ICESI, Facultad de Ciencias de la Salud. Cali, Colombia.

${ }^{3}$ Departamento de Reumatología, Fundación Valle del Lili. Cali, Colombia.

Introducción: la hipofisitis por inmunoglobulina G 4 (IgG4) es una condición infrecuente, consiste en un compromiso fibroinflamatorio usualmente crónico de la glándula hipófisis. No hay datos exactos de la prevalencia, aunque se estima anualmente un caso por cada 9 millones de personas. Se documenta el caso de una mujer joven con manifestaciones neurológicas asociadas con esta patología, confirmada por biopsia e inmunohistoquímica.

Objetivo: presentar el caso de una paciente con diagnóstico de hipofisitis por IgG4.

Métodos: se realizó la revisión de la historia clínica, análisis de información y confrontación con literatura reportada.

Resultados: paciente de 47 años, sin antecedentes patológicos previos, inició con cefalea de 3 meses de evolución, acompañada de diplopía y fotofobia. La resonancia magnética nuclear cerebral documentó lesión selar con un componente supraselar, con infundíbulo desplazado hacia la parte posterior, de macroadenoma quístico. El perfil hormonal era normal. En el postoperatorio resultó sin mayores complicaciones. Se llevó a resección por el abordaje endonasal, el estudio histopatológico con zonas de fibrosis, infiltrado de predominio plasmolinfocitario y con IgG4 positiva, lo que configura una enfermedad esclerosante relacionada con IgG4. Después de la resección y manejo con esteroides hubo una resolución de los síntomas.

Discusión: la enfermedad por IgG4 es una condición sistémica, la entidad tiene un curso clínico silente, con manifestaciones que varían dependiendo de la localización y, en la mayoría de los casos, iniciando con síntomas compresivos y obstructivos. El compromiso del sistema nervioso central es extremadamente infrecuente, aunque en este la presentación hipofisaria es la más común; se caracteriza por hipopituitarismo, diabetes insípida o efecto de masa local sintomática, como fue el caso de la paciente. 
Póster

\section{Hiponatremia por insuficiencia suprarrenal secundaria. Una serie de casos de un hospital universitario de tercer nivel en Pereira, Colombia}

\author{
Guzmán ES, Giraldo J, Medina DA, Forero JE, \\ Alzate JA, Vallejo S.
}

Hospital Universitario San Jorge. Pereira, Colombia.

Antecedentes: la hiponatremia por insuficiencia suprarrenal secundaria con frecuencia se subestima y se diagnostica de manera insuficiente.

Objetivo: resaltar la importancia de una hiponatremia suprarrenal secundaria como causa de hiponatremia euvolémica para evitar un tratamiento y diagnóstico inapropiados.

Métodos: se describió una serie de casos de 13 pacientes con hiponatremia euvolémica atribuible a insuficiencia suprarrenal secundaria con sus características clínicas, bioquímicas y radiológicas, y se comparó con informes similares publicados previamente.

Hallazgos: todos los pacientes con insuficiencia suprarrenal secundaria presentaron hiponatremia euvolémica hipotónica. El 53,8 \% de los pacientes era de sexo femenino con una mediana de edad de 52 años. La concentración media de cortisol fue de 2,9 $\mu \mathrm{g} / \mathrm{dL}$ (rango intercuartílico [IQR] 1,8-3,34 $\mu \mathrm{g} / \mathrm{dL}$ ) y la concentración media de corticotropina (ACTH) fue de 6,65 pg/nL (IQR 4,1-9,2 pg/nL). Todos los pacientes tenían una alta densidad urinaria y características indistinguibles de síndrome de secreción inadecuada de la. hormona antidiurética (SIADH). El hipogonadismo hipogonadotrópico y el hipotiroidismo central fueron los ejes hipofisarios afectados más comúnmente acompañados. La hipoglucemia, la hipotensión y la hiperpotasemia fueron hallazgos infrecuentes en estos pacientes. La etiología más frecuente identificada fue el síndrome de la silla turca vacía.

Conclusiones: la hiponatremia hipotónica euvolémica es la presentación principal de la insuficiencia suprarrenal secundaria y, con frecuencia, no se acompaña de otras manifestaciones de deficiencia de glucocorticoides. Esta enfermedad es clínica y bioquímica indistinguible de SIADH. Un umbral de sospecha bajo y una medición sérica de cortisol por la mañana en estos pacientes es esencial para evitar un diagnóstico y manejo inapropiados.
Póster

\section{Linfoma de células B grande difuso que se presentó con infiltración hipofisaria, hipopituitarismo y compromiso del nervio oculomotor. Reporte de caso}

\author{
Arias D, Revelo S, Forero JE, Vallejo S.
}

Universidad Tecnológica de Pereira. Pereira, Colombia.

Introducción: el linfoma es un tumor sólido del sistema inmune. Es un grupo heterogéneo de neoplasias que tiene una presentación clínica variable, especialmente en el caso de los linfomas no Hodgkin. El compromiso del sistema nervioso y de la hipófisis es poco frecuente.

Metodología: reporte de caso.

Presentación de caso: mujer de 55 años que consultó por adenomegalias generalizadas, astenia, adinamia, somnolencia y diplopía; sin síntomas de linfoma de células B. El examen físico reveló una parálisis incompleta del nervio oculomotor (par III) derecho. No había hepatoesplenomegalia. Una resonancia magnética nuclear (RMN) cerebral mostró un crecimiento difuso de la hipófisis con un realce heterogéneo después de la administración del contraste. Una biopsia del ganglio cervical confirmó la presencia de linfoma de células B grande difuso. El laboratorio inicial reveló una hiponatremia normovolémica con sodio y osmolaridad urinaria elevados, probablemente en relación con un síndrome de secreción inadecuada de la hormona antidiurética (SIADH). El estudio hormonal reveló una tirotropina (TSH) suprimida con T4L normal y T3T disminuida, el cortisol sérico fue de 12,1 $\mu \mathrm{g} / \mathrm{dL}$ en presencia de una corticotropina (ACTH) 12,3 pg/mL (rango de referencia: 12-60); tenía una Hormona foliculoestimulante (FSH) y un Factor de crecimiento insulínico tipo 1 (IGF-1) disminuidos; la prolactina (PRL) fue normal. Basado en estos hallazgos se planteó el diagnóstico de hipopituitarismo secundario a infiltración hipofisaria por linfoma. Infortunadamente, durante la hospitalización la paciente desarrolló una sepsis de origen respiratorio y falleció después de varios días de tratamiento en la unidad de cuidados intensivos (UCI).

Discusión y conclusiones: el compromiso hipofisario por linfoma es infrecuente. En este caso, el hallazgo de hiponatremia normovolémica motivó el estudio del eje tirótropo y adrenal que permitió detectar el compromiso hormonal sugestivo de hipopituitarismo. 
Póster

Supervivencia de pacientes con tumores neuroendocrinos en un hospital de tercer nivel, 2012-2017

\author{
Pinzón A, Jiménez CE, Mondragón AE, Gutiérrez Y, \\ García HA.
}

Hospital Universitario Hernando Moncaleano Perdomo (HUHMP). Neiva, Colombia.

Introducción: la incidencia de tumores neuroendocrinos (TNE) ha venido aumentado notablemente de 2,48 hasta 5,86 en los últimos 15 años. En Colombia los estudios en esta área son escasos.

Objetivo: describir la supervivencia, las características sociodemográficas y las principales manifestaciones clínicas de los TNE.

Diseño: estudio observacional y descriptivo. Se obtuvo una base de datos de 55 pacientes diagnosticados con TNE desde el 2012 al 2017. Los datos se procesaron en el programa estadístico STATA versión 13 y el análisis de supervivencia se realizó a través del método Kaplan Meier.

Resultados: de los pacientes con diagnóstico de TNE, el 64 $\%$ fue de sexo masculino. La edad media fue 57,5 años (rango: 7-82). La procedencia fue urbana en el $82 \%$, la mayoría de Neiva con un 38 \%, seguido de Garzón con un 11\%.

Distribución: TNE pulmonares (23/55), tumores endocrinos gastroenteropancreáticos (NTE-GEP) (22/55) y otros (10/55). Los signos y síntomas más reportados de los tumores pulmonares fueron tos con un 44,4 \% y disnea con un 44,4 \%; de los TNE-GEP fueron emesis con un $21 \%$ y dolor abdominal con un $78 \%$.

El $52 \%$ de los pacientes falleció en el primer año. La neoplasia que más fallecidos reportó fue el carcinoma pulmonar de células pequeñas (26,92\%), seguido de carcinoma de célula grande (19,23\%). La función de supervivencia al $42 \%$ fue en el día 52 para los pacientes con TNE-GEP; para los TNE pulmonares, la función de supervivencia al 50 \% se encuentra al día 52.

Conclusiones: los pacientes con TNE del HUHMP presentan una función de supervivencia menor que en la literatura.

\section{Pegvisomant, un último recurso en acromegalia no respondedora al manejo medicoquirúrgico inicial: reporte de caso}

Sánchez PE, Rojas W, Tovar H.

Fundación Universitaria de Ciencias de la Salud, Hospital de San José. Bogotá D. C., Colombia.
$\mathrm{L}$ a acromegalia es una enfermedad caracterizada por la sobreproducción de la hormona del crecimiento (GH) y el factor de crecimiento insulinoide de tipo 1 (IGF-1; somatomedina C) luego del cierre de la placa epifisaria. El 95 $\%$ de los casos es secundario a tumores hipofisarios con hipersecreción de GH desde las células somatotropas, la mayoría es de origen esporádico y $5 \%$ familiar. El resto puede ser secundario a la producción excesiva de hormona liberadora de hormona del crecimiento (GHRH) por otros tumores.

Con una prevalencia de 86-240 casos/millón, igual en ambos sexos, la edad diagnóstica promedio es de 40-50 años con un retraso diagnóstico de hasta 10 años. El objetivo es lograr niveles de GH $<1 \mu \mathrm{g} / \mathrm{L}$ e IGF-1 normales para la edad. El tratamiento inicial es la resección quirúrgica de la lesión, asociada en casos refractarios con terapia médica con análogos de somatostatina (SRL), antagonistas de los receptores de GH, agonistas dopaminérgicos y radioterapia.

Se presenta a una paciente con acromegalia secundaria a macroadenoma hipofisario productor de GH llevada a resección quirúrgica transesfenoidal, con hipopituitarismo secundario. En el seguimiento imagenológico se documentó persistencia de la lesión y progresión de los síntomas, por lo cual se indicó el manejo con octreótido $30 \mathrm{mg}$ q30d y radioterapia convencional ante la imposibilidad de un nuevo procedimiento quirúrgico debido al compromiso vascular. Los niveles posteriores de GH e IGF-1 estuvieron fuera de las metas, por lo cual se asoció el antagonista de los receptores de IGF-1 (pegvisomant) 10 mg subcutáneo (SC) q24h con transaminitis inicial, por lo cual se titularon dosis a q48h; luego de 10 años de radioterapia y 1 año sin pegvisomant, con octreótido cada 60 días tiene control clínico y bioquímico de la acromegalia.

\section{Póster}

Prolactinoma abscedado: absceso hipofisario asociado a un macroadenoma productor de prolactina. Importancia clínica y diagnóstica de las secuencias de resonancia en lesiones selares. Reporte de caso

Torres JL, Vargas MA, Coronel N, Aristizábal N.

Clínica Las Américas. Medellín, Colombia.

Revista Colombiana de Endocrinología, Diabetes y Metabolismo 
$\mathrm{L}$ os abscesos hipofisarios son condiciones clínicas extremadamente infrecuentes en la práctica diaria; pueden aparecer como lesiones hipofisarias primarias (sobre una glándula sana) o ser secundarios a craneofaringiomas, quistes de Rathke o a un adenoma. Es de suma importancia la evaluación clínica oportuna de esta entidad para ofrecer un tratamiento dirigido acertado y, de este modo, evitar la alta mortalidad asociada. Para el 2018 en la literatura médica internacional se habían reportado solo 23 casos de adenomas hipofisarios con infección secundaria y, en términos generales, los abscesos constituyen entre el 0,27 \% y 0,60 \% de todas las lesiones pituitarias.

Se presenta el caso de un paciente joven de sexo masculino con un macroadenoma productor de prolactina, quien había iniciado un tratamiento con un agonista dopa con buena respuesta, pero presentó una cefalea intensa y empeoramiento de su estado general de forma súbita, por lo que se realizaron estudios en los que finalmente se encontró un absceso hipofisario asociado. El objetivo de este reporte es enfatizar en sus características clínicas e imagenológicas y además presentar un algoritmo diagnóstico diferencial basado en las características imagenológicas de las diferentes patologías selares con el fin de identificar rápidamente este tipo de lesiones que ponen en riesgo la vida y requieren intervención quirúrgica temprana.

\section{Póster}

\section{Síndrome de Cushing de difícil diagnóstico secundario al uso de un "producto naturista" para los dolores articulares y musculares}

Fierro $L F^{1}$, González $A M^{1,2}$, Álvarez $M A^{2}$, Moreno $G A^{2}$, Rodríguez $S^{2}$, Tarquino $D^{2}$.

${ }^{I}$ Instituto Nacional de Cancerología.

${ }^{2}$ Universidad Nacional de Colombia.

Introducción: el síndrome de Cushing es un grupo de signos y síntomas causados por la exposición crónica al exceso de glucocorticoides. El Cushing de origen endógeno es un reto diagnóstico, pero lo es más cuando no se descarta la exposición a glucocorticoides exógenos, ahora presentes de forma oculta en productos vendidos como de origen natural para el control del dolor.

Presentación del caso: se describe el caso clínico de un paciente de 40 años con artritis psoriásica diagnosticada a los 30 años aproximadamente, quien a los 39 años se le diagnosticó diabetes mellitus de difícil manejo pese a recibir dosis altas de insulina, hipertensión arterial no controlada con el uso de 3 antihipertensivos y signos clínicos típicos del síndrome de Cushing (obesidad central, fascias en luna llena) quien negó haber consumido glucocorticoides. Se le practicaron pruebas diagnósticas para confirmar hipercortisolismo con resultados discordantes y variables. Solo después de muchos interrogatorios se logró conocer que el paciente consumía de forma crónica un producto naturista conocido como artrin, que fue analizado en el laboratorio de la institución con resultado positivo para cortisol. Se realizó el cambio a prednisolona para el retiro gradual y se logró la reversión de la diabetes mellitus y de los cambios físicos.

Conclusión: la exposición crónica a glucocorticoides exógenos, ya sea subrepticia, desconocida o prescrita, causa síndrome de Cushing y debe distinguirse tempranamente de las formas endógenas para evitar la realización de pruebas diagnósticas y tratamientos inadecuados.

Palabras clave: síndrome de Cushing, glucocorticoides exógenos, suplementos herbales.

\section{Póster}

\section{Síndrome de Cushing, comportamiento posterior al tratamiento}

\author{
Salgado CA, Muñoz JP, Bedoya V, Osorio CV, Tabares \\ AA, Abreu A.
}

Programa de Medicina Interna, Universidad Libre, Seccional Cali; Centro médico Imbanaco. Cali, Colombia.

Introducción: el síndrome de Cushing es una patología de prevalencia incierta en la ciudad de Cali, con una gran carga mórbida al momento de su presentación. La respuesta a los manejos estandarizados es variable. Es necesario determinar la respuesta clínica a diferentes opciones de tratamiento ofrecidas.

Diseño: estudio de cohorte retrospectiva, realizado entre el 1 de enero de 2008 hasta el 31 de diciembre de 2017.

Métodos: se incluyeron pacientes con diagnóstico de síndrome de Cushing, cuyo control se realizaba en la consulta endocrinológica de un centro de salud nivel IV de la ciudad de Cali. Se revisaron las variables demográficas, presencia de comorbilidades asociadas, persistencia, remisión y recurrencia de la enfermedad.

Resultados: se incluyó a un total de 45 pacientes cuya edad promedio fue de 46 años, con un $60 \%$ de pacientes femeninos y el 47 \% tenía hipertensión. Todos los pacientes fueron llevados a cirugía en su primer abordaje. Se presentó la remisión clínica en el 49 \% de la población, con recurrencia 
del $32 \%$. En el análisis de subgrupos, los pacientes con enfermedad de Cushing presentaron una remisión del $41 \%$, con una recurrencia del $38 \%$; mientras que los pacientes con adenoma suprarrenal presentaron una remisión del $100 \%$ con una recurrencia de $25 \%$. Por su parte, de los 5 pacientes con tumores ectópicos productores de corticotropina (ACTH) solo
1 remitió, aunque este único paciente presentó una recurrencia posterior a la remisión.

Conclusiones: la eficacia de las diversas terapias disponibles varía según las poblaciones estudiadas. Las variables demográficas son similares a los reportes de la literatura. Se evidenció un menor porcentaje de comorbilidades asociadas. 